\title{
THE VALUE OF DIAGNOSTIC TESTS IN PREDICTING HYPERBILIRUBINEMIA IN ABO INCOMPATIBILITY
}

\author{
D. Stoniene, J. Buinauskiene, E. Markuniene, R. Tameliene, A. Kudreviciene \\ Hospital of Lithuanian University Health Sciences, Kaunas, Lithuania
}

\begin{abstract}
Aim of the study: To establish predictive criteria of neonatal hyperbilirubinemia in ABO incompatibility.
\end{abstract}
Method: 668 healthy full-term newborns with ABO incompatibility were initially enrolled in the prospective cohort study.

Results: Hyperbilirubinemia was diagnosed to 71(10.6 \%) newborns. Positive direct Coombs' test (DCT) was diagnosed significantly more often to newborns with hyperbilirubinemia, even $85.5 \%$ newborns of this group had negative DCT. Total serum bilirubin (TSB) level cut-off point at the 6 hour of life was 76.0 $\mu \mathrm{mol} / \mathrm{L}$ (sensitivity 0.9 , specificity 0.9$)$ i.e. $9.7 \%$ in healthy group and $87.3 \%(\mathrm{P}<0.001)$ of newborns with hyperbilirubinemia had TSB $76.0 \mu \mathrm{mol} / \mathrm{L}$. According to the determined value, it can be predicted that newborns with TSB level $>76 \mu \mathrm{mol} / \mathrm{L}$ at the age of 6 hours will have hyperbilirubinemia with an odds ratio of $64.0[30.2-135.5]$. With a ROC curve hemocrit cut-off point $44 \%$ was determined (sensitivity 0.5 ; speificity 0.5 ). The cut-off point of reticulocytes was determined to be $4.8 \%$ (sensitivity 0.8 , specificity 0.7 ). Binary logistic regression analysis model allows to predict that newborns will have a higher odds ratio to be diagnosed with hyperbilirubinemia (47.5[20.4-110.4]), when TSB at the 6 hour of life is $>76 \mu \mathrm{mol} / \mathrm{L}$, $3.0[1.4-6.4]$, reticulocytes $>4.8 \%, 6.0[2.8-12.7]$, hematocrit $<44 \%, 2.6[1.0-7.3]$, spehrocytes $>1.5$ (units), positive DCT (1.7[0.6-4.8]).

Conclusions: The most significant factor in predicting ABO hyperbilirubinemia is TSB at the age of 6 hours $>76 \mu \mathrm{mol} / \mathrm{L}$. The prognostic value of the hematocrit and reticulocytes level in blood was lower. The number of spherocytes in blood and DCT has no prognostic value. 